\title{
Long-term effects of fenofibrate on VLDL and HDL subspecies in participants with type 2 diabetes mellitus
}

\author{
A. Hiukka • E. Leinonen • M. Jauhiainen • J. Sundvall • \\ C. Ehnholm • A. C. Keech • M. R. Taskinen
}

Published online: 10 October 2007

(C) Springer-Verlag 2007

An incorrect conversion factor was used by the authors resulting in values given in $\mathrm{g} / \mathrm{l}$ being ten times higher than they should have been. The corrected sections of Tables 1 , 2, 3, 4 are reproduced below.

In addition there are two text corrections:

The last paragraph of the Abstract should have read:

Conclusions/interpretation Fenofibrate markedly reduced large VLDL particles and produced a clear shift in HDL subspecies towards smaller particles. The HDL3-C increase in conjunction with unchanged apoA-I levels is a dilemma with regard to cardiovascular disease.

The last sentence of the subsection on 'Impact of homocysteine levels on HDL' in the Results should have read:

In patients with low homocysteine levels ( $n=42)$, HDL-C and apoA-I levels were slightly increased by fenofibrate ( $\Delta=0.05 \mathrm{mmol} / 1$ for HDL-C and $\Delta=0.034 \mathrm{~g} / \mathrm{l}$ for apoA-I), whereas in patients with high homocysteine levels $(n=42)$ HDL-C and apoA-I levels decreased $(\Delta=-0.04 \mathrm{mmol} / \mathrm{l}$ for HDL-C and $\Delta=-0.014 \mathrm{~g} / 1$ for apoA-I) $(p=0.028$ for both the differences in $\triangle$ HDL-C and $\triangle$ apoA-I between the low and high homocysteine groups).

The online version of the original article can be found at: http://dx.doi. org/10.1007/s00125-007-0751-8.

\footnotetext{
A. Hiukka $\cdot$ E. Leinonen $\cdot$ M. R. Taskinen $(\bowtie)$

Department of Medicine, Division of Cardiology,

Helsinki University Hospital and Biomedicum,

Haartmaninkatu 8,

00290 Helsinki, Finland

e-mail: Marja-Riitta.Taskinen@helsinki.fi

M. Jauhiainen · C. Ehnholm

Department of Molecular Medicine,

National Public Health Institute and Biomedicum,

Helsinki, Finland

J. Sundvall

Department of Health and Functional Capacity,

National Public Health Institute,

Helsinki, Finland

A. C. Keech

NHMRC Clinical Trials Centre, University of Sydney,

Sydney, NSW, Australia
} 
Table 1 Basic lipid panel and lipid transfer protein activities before and during Fenofibrate treatment

Baseline

Fifth year

Difference of change between the groups

$\operatorname{apoB}(\mathrm{g} / \mathrm{l})$

Placebo

Fenofibrate

$0.96(0.82-1.07)$

$1.00(0.89-1.17)$

$1.00(0.91-1.12)$

$0.84(0.70-0.98)$

$-24.7(-18.4,-31.0)$

$<0.001$

Table 2 Triacylglycerol, cholesterol and particle mass concentration of VLDL 1, VLDL 2 and IDL in type 2 diabetic patients

\begin{tabular}{|c|c|c|c|c|c|c|}
\hline & \multirow[t]{2}{*}{ Baseline } & \multicolumn{2}{|l|}{ Second year } & \multicolumn{2}{|l|}{ Fifth year } & \multirow[t]{2}{*}{$p$ value } \\
\hline & & & $\begin{array}{l}\text { Difference of change } \\
\text { between the groups }\end{array}$ & & $\begin{array}{l}\text { Difference of change } \\
\text { between the groups }\end{array}$ & \\
\hline \multicolumn{7}{|c|}{ VLDL1 particle mass (g/l) } \\
\hline Placebo & $0.88(0.50-1.44)$ & $0.85(0.51-1.20)$ & \multirow[t]{2}{*}{$-39.4(-25.6,-53.6)$} & $0.81(0.51-1.26)$ & \multirow[t]{2}{*}{$-43.5(-29.5,-59.4)$} & \multirow[t]{2}{*}{$<0.001$} \\
\hline Fenofibrate & $0.85(0.49-1.42)$ & $0.47(0.25-0.73)$ & & $0.38(0.22-0.85)$ & & \\
\hline \multicolumn{7}{|c|}{ VLDL2 particle mass $(\mathrm{g} / \mathrm{l})$} \\
\hline Placebo & $0.60(0.42-0.78)$ & $0.60(0.41-0.80)$ & \multirow[t]{2}{*}{$-32.1(-19.2,-44.8)$} & $0.54(0.42-0.80)$ & \multirow[t]{2}{*}{$-32.5(-20.0,-45.3)$} & \multirow[t]{2}{*}{$<0.001$} \\
\hline Fenofibrate & $0.58(0.39-0.78)$ & $0.36(0.24-0.52)$ & & $0.38(0.23-0.60)$ & & \\
\hline \multicolumn{7}{|c|}{ IDL particle mass $(\mathrm{g} / \mathrm{l})$} \\
\hline Placebo & $0.39(0.30-0.50)$ & $0.39(0.30-0.46)$ & \multirow[t]{2}{*}{$-5.7(2.6,-14.5)$} & $0.40(0.32-0.47)$ & \multirow[t]{2}{*}{$-12.0(-2.0,-21.5)$} & \multirow[t]{2}{*}{0.019} \\
\hline Fenofibrate & $0.38(0.30-0.49)$ & $0.33(0.26-0.43)$ & & $0.32(0.25-0.46)$ & & \\
\hline
\end{tabular}

Table 3 Cholesterol concentration, particle mass of HDL2 and HDL3, and concentrations of apolipoproteins and apoA-containing lipoproteins in type 2 diabetic patients

\begin{tabular}{|c|c|c|c|c|c|c|}
\hline & \multirow[t]{2}{*}{ Baseline } & \multicolumn{2}{|l|}{ Second year } & \multicolumn{2}{|l|}{ Fifth year } & \multirow[t]{2}{*}{$p$ value } \\
\hline & & & $\begin{array}{l}\text { Difference of change } \\
\text { between the groups }\end{array}$ & & $\begin{array}{l}\text { Difference of change } \\
\text { between the groups }\end{array}$ & \\
\hline \multicolumn{7}{|c|}{ HDL2 Particle mass (g/l) } \\
\hline Placebo & $0.89(0.72-1.20)$ & $0.89(0.71-1.22)$ & \multirow[t]{2}{*}{$-20.0(-12.5,-28.0)$} & $0.97(0.71-1.41)$ & \multirow[t]{2}{*}{$-23.1(-13.6,-32.2)$} & \multirow[t]{2}{*}{$<0.001$} \\
\hline Fenofibrate & $0.95(0.72-1.29)$ & $0.73(0.55-0.96)$ & & $0.76(0.61-1.02)$ & & \\
\hline \multicolumn{7}{|c|}{ HDL3 Particle mass $(\mathrm{g} / \mathrm{l})$} \\
\hline Placebo & $2.13(1.93-2.35)$ & $2.32(2.16-2.58)$ & \multirow[t]{2}{*}{$7.7(2.8,12.8)$} & $2.41(2.26-2.65)$ & \multirow[t]{2}{*}{$12.5(7.2,17.9)$} & \multirow[t]{2}{*}{$<0.001$} \\
\hline $\begin{array}{l}\text { Fenofibrate } \\
\text { apoA-I (g/l) }\end{array}$ & $2.14(1.92-2.28)$ & $2.50(2.24-2.71)$ & & $2.69(2.44-2.94)$ & & \\
\hline Placebo & $1.34(1.21-1.51)$ & $1.32(1.21-1.48)$ & \multirow[t]{2}{*}{$-0.7(-4.2,2.8)$} & $1.34(1.23-1.51)$ & \multirow[t]{2}{*}{$-0.5(-3.9,3.7)$} & \multirow[t]{2}{*}{ NS } \\
\hline $\begin{array}{l}\text { Fenofibrate } \\
\text { apoA-II }(\mathrm{g} / \mathrm{l})\end{array}$ & $1.36(1.25-1.45)$ & $1.32(1.21-1.46)$ & & $1.34(1.25-1.50)$ & & \\
\hline Placebo & $0.34(0.31-0.37)$ & $0.34(0.31-0.39)$ & \multirow[t]{2}{*}{$22.9(18.2,28.1)$} & $0.31(0.27-0.37)$ & \multirow[t]{2}{*}{$26.9(19.6,34.4)$} & \multirow[t]{2}{*}{$<0.001$} \\
\hline Fenofibrate & $0.33(0.30-0.37)$ & $0.40(0.37-0.46)$ & & $0.42(0.35-0.48)$ & & \\
\hline \multicolumn{7}{|l|}{ LpA-I (g/l) } \\
\hline Placebo & $0.48(0.41-0.61)$ & $0.46(0.37-0.59)$ & \multirow[t]{2}{*}{$-20.4(-13.4,-27.5)$} & $0.48(0.39-0.65)$ & \multirow[t]{2}{*}{$-21.1(-12.7,-30.0)$} & \multirow[t]{2}{*}{$<0.001$} \\
\hline Fenofibrate & $0.49(0.41-0.59)$ & $0.35(0.30-0.42)$ & & $0.39(0.31-0.45)$ & & \\
\hline \multicolumn{7}{|l|}{ LpAI-AII (g/l) } \\
\hline Placebo & $0.83(0.75-0.90)$ & $0.86(0.78-0.95)$ & \multirow[t]{2}{*}{$10.6(5.5,16.0)$} & $0.83(0.77-0.96)$ & \multirow[t]{2}{*}{$11.5(5.3,17.3)$} & \multirow[t]{2}{*}{$<0.001$} \\
\hline Fenofibrate & $0.83(0.77-0.92)$ & $0.96(0.89-1.06)$ & & $0.97(0.88-1.07)$ & & \\
\hline
\end{tabular}


Table 4 The baseline HDL panel in the groups of low and high homocysteine levels

Lowest quartile $(n=56)$

$1.37(1.21-1.55)$

$0.34(0.30-0.38)$

$0.51(0.41-0.65)$

$0.85(0.79-0.93)$
Highest quartile $(n=60)$

$p$ value

apoA-I (g/l)

apoA-II $(\mathrm{g} / \mathrm{l})$

LpA-I (g/l)

LpAI-AII (g/l)
$1.29(1.19-1.43)$

$0.32(0.31-0.36)$

$0.48(0.41-0.61)$

$0.81(0.74-0.87)$
0.131

0.040

0.904

0.017 